PROCEEDINGS OF THE

AMERICAN MATHEMATICAL SOCIETY

Volume 135, Number 4, April 2007, Pages 969-975

S 0002-9939(06)08558-3

Article electronically published on September 26, 2006

\title{
ON A PROBLEM OF D. H. LEHMER
}

\author{
STÉPHANE R. LOUBOUTIN, JOËL RIVAT, AND ANDRÁS SÁRKÖZY
}

(Communicated by Wen-Ching Winnie Li)

\begin{abstract}
Let $p$ be an odd prime number. For $n \in\{1, \ldots, p-1\}$ we denote the inverse of $n$ modulo $p$ by $n^{*}$ with $n^{*} \in\{1, \ldots, p-1\}$. Given $\varepsilon>0$, we prove that in any range $n \in\{N+1, \ldots, N+L\} \subseteq\{1, \ldots, p-1\}$ of length $L \geq p^{1 / 2+\varepsilon}$ the probability that $n^{*}$ has the same parity as $n$ tends to $1 / 2$ as $p \rightarrow+\infty$. This result was previously known only to hold true in the full range $n \in\{1, \ldots, p-1\}$ of length $L=p-1$. We will also obtain quantitative results on the pseudorandomness of the sequence $(-1)^{n+n^{*}}$ for which we estimate the well-distribution $W$ and correlation measures $C_{k}$ as defined by Mauduit and Sárközy (1997).
\end{abstract}

\section{INTRODUCTION}

The aim of this paper is to use a construction proposed by D. H. Lehmer (See R. Guy [5, pp. 139-140]) to give new examples of sequences with good pseudorandom properties. Let $p$ be an odd prime number. For $n \in\{1, \ldots, p-1\}$ we denote the inverse of $n$ modulo $p$ by $n^{*}$ with $n^{*} \in\{1, \ldots, p-1\}$. By convention we fix $0^{*}=0$. Let $E_{p}=\left(e_{1}, \ldots, e_{p-1}\right)$ defined by

$$
e_{n}=(-1)^{n+n^{*}}= \begin{cases}+1 & \text { if } n^{*} \equiv n \bmod 2, \\ -1 & \text { if } n^{*} \neq \equiv n \bmod 2 .\end{cases}
$$

Z. Wenpeng [11, Theorem 1] and [12, Corollary, p. 48] (see also [13, 14, 15, 16]) has proved that

$$
\left|\sum_{1 \leq n \leq p-1} e_{n}\right| \ll p^{1 / 2} \log ^{2} p .
$$

Roughly speaking, this means that half of the $p-1$ integers $n \in\{1, \ldots, p-1\}$ satisfy $n^{*} \equiv n \bmod 2\left(\right.$ in which case $\left.e_{n}=+1\right)$ while the other half satisfy $n^{*} \not \equiv n \bmod 2$ $\left(e_{n}=-1\right.$ ). However, (2) does not prevent the $e_{n}$ 's from all being equal to +1 (or to -1$)$ for long strings of consecutive integers, say of length $(p-1) / 2$. Our first result generalizes estimate (2) to short sums, thus proving that the $e_{n}$ 's cannot all be equal to +1 (or to -1 ) for strings of consecutive integers of length $\gg p^{1 / 2} \log ^{2} p$.

Received by the editors August 8, 2005 and, in revised form, November 8, 2005.

2000 Mathematics Subject Classification. Primary 11K45; Secondary 11L05, 11L40.

Key words and phrases. Pseudo-random, binary sequence, correlation.

The research of the third author was partially supported by the Hungarian National Foundation for Scientific Research, Grants No T043623 and T049693. This paper was written when the third author was visiting the Institut de Mathématiques de Luminy.

(C)2006 American Mathematical Society 
Theorem 1. For $1 \leq M<N<p$, we have

$$
\left|\sum_{M \leq n \leq N} e_{n}\right| \leq \frac{8}{\pi^{2}} p^{1 / 2} \log ^{2}(5 p)+2 .
$$

Not only is our bound (3) much more general than (2), but our proof is elementary and much simpler than Z. Wenpeng's proof of (2).

In a series of papers, partly with further co-authors, we studied finite pseudorandom binary sequences

$$
E_{N}=\left\{e_{1}, \ldots, e_{N}\right\} \in\{-1,+1\}^{N} .
$$

Our goal was to develop a constructive theory of pseudorandomness, i.e., to introduce and study measures of pseudorandomness which cover pseudorandom properties important in applications and which can be handled and estimated at least for large families of binary sequences. Indeed, in 6] Mauduit and Sárközy introduced the following measures of pseudorandomness: the well-distribution measure of $E_{N}$ is defined by

$$
W\left(E_{N}\right)=\max _{a, b, t}\left|\sum_{j=0}^{t-1} e_{a+j b}\right|,
$$

where the maximum is taken over all $a, b, t \in \mathbf{N}$ such that $1 \leq a \leq a+(t-1) b \leq N$, and the correlation measure of order $k$ of $E_{N}$ is defined as

$$
C_{k}\left(E_{N}\right)=\max _{M, D}\left|\sum_{n=1}^{M} e_{n+d_{1}} e_{n+d_{2}} \cdots e_{n+d_{k}}\right|
$$

where the maximum is taken over all $D=\left(d_{1}, \ldots, d_{k}\right)$ and $M$ such that $0 \leq d_{1}<$ $\cdots<d_{k} \leq N-M$. Then the sequence is considered as a "good" pseudorandom sequence if both these measures $W\left(E_{N}\right)$ and $C_{k}\left(E_{N}\right)$ (at least for small $k$ ) are "small" in terms of $N$ (in particular both are $o(N)$ as $N \rightarrow \infty$ ). Notice that for almost all $E_{N} \in\{-1,+1\}^{N}$, both $W\left(E_{N}\right)$ and $C_{k}\left(E_{N}\right)$ are less than $N^{1 / 2}(\log N)^{c}$ (see [2] and 1]). One might like to know whether the sequence $\left(e_{n}\right)$ defined by (1) is such a "good" pseudorandom sequence. We shall, in fact, answer this question for a family of sequences more general than (11). Let $f(x) \in \mathbf{F}_{p}[x]$. We identify the field $\mathbf{F}_{p}$ and the set $\{0, \ldots, p-1\}$ and for $n \in \mathbf{Z}$ we assume that $f(n) \in\{0, \ldots, p-1\}$. (These details are important in order to further reduce modulo 2.) Under these conditions we may generalize construction (10) as follows:

$$
e_{n}= \begin{cases}+1 & \text { if } f(n)^{*} \equiv f(n) \bmod 2, \\ -1 & \text { if } f(n)^{*} \not \equiv f(n) \bmod 2 .\end{cases}
$$

Theorem 2. Let $f(x) \in \mathbf{F}_{p}[x]$ of degree $d$ with $p>d \geq 1$, let $s$ be its number of distinct roots in an algebraic closure $\overline{\mathbf{F}_{p}}$ of $\mathbf{F}_{p}$, and let $E_{p}=\left(e_{1}, \ldots, e_{p-1}\right)$ defined by (4). Then,

$$
\begin{aligned}
W\left(E_{p}\right) & \ll(d+s) p^{1 / 2} \log ^{3} p, \\
C_{2}\left(E_{p}\right) & \ll(d+s) p^{1 / 2} \log ^{5} p,
\end{aligned}
$$

where the implied constants are absolute. 


\section{Proof of Theorem 1}

\subsection{Exponential sums.}

Lemma 3. Let $A, B, m \in \mathbf{Z}_{\geq 0}$ such that $m$ is odd and $0 \leq A<B \leq(m-1) / 2$. Then, for $1 \leq n \leq m-1$, we have

$$
(-1)^{n} \mathbf{1}_{[2 A+1,2 B]}(n)=\frac{1}{m} \sum_{1 \leq|h| \leq(m-1) / 2} v_{m}(h ; A, B) \exp (2 \pi i h n / m),
$$

where

$$
\begin{aligned}
v_{m}(h ; A, B) & =(1-\exp (2 \pi i h / m)) \sum_{A+1 \leq k \leq B} \exp (-4 \pi i h k / m) \\
& =-i \frac{\sin (2 \pi h(B-A) / m)}{\cos (\pi h / m)} \exp (2 \pi i h(A+B+1 / 2) / m)
\end{aligned}
$$

satisfies

$$
\sum_{|h| \leq(m-1) / 2}\left|v_{m}(h ; A, B)\right| \leq \frac{2}{\pi} m \log (5 m) .
$$

Proof. For odd $m \geq 3$ and $1 \leq n \leq(m-1) / 2$, we have

$$
\begin{aligned}
& (-1)^{n} \mathbf{1}_{[2 A+1,2 B]}(n)=\sum_{A+1 \leq k \leq B}\left(\mathbf{1}_{\{2 k\}}(n)-\mathbf{1}_{\{2 k-1\}}(n)\right) \\
& =\sum_{A+1 \leq k \leq B} \frac{1}{m} \sum_{|h| \leq(m-1) / 2}\left(\exp \left(\frac{2 \pi i h(n-2 k)}{m}\right)-\exp \left(\frac{2 \pi i h(n-(2 k-1))}{m}\right)\right) \\
& =\frac{1}{m} \sum_{|h| \leq(m-1) / 2} v_{m}(h ; A, B) \exp (2 \pi i h n / m) .
\end{aligned}
$$

Observe that $v_{m}(0 ; A, B)=0$, and this completes the proof of (7). To prove (8), we write

$$
\sum_{|h| \leq(m-1) / 2}\left|v_{m}(h ; A, B)\right| \leq 2 \sum_{1 \leq h \leq(m-1) / 2} \frac{1}{\cos (\pi h / m)}:=2 S(m) .
$$

By the convexity of the function $t \mapsto 1 / \sin (\pi t / m)$, we get

$$
\begin{aligned}
S(m) & =\sum_{1 \leq k \leq(m-1) / 2} \frac{1}{\sin \left(\frac{\pi}{m}\left(k-\frac{1}{2}\right)\right)} \leq \frac{1}{\sin \left(\frac{\pi}{2 m}\right)}+\int_{3 / 2}^{m / 2} \frac{d t}{\sin \left(\frac{\pi}{m}\left(t-\frac{1}{2}\right)\right)} \\
& \leq \frac{1}{\sin \left(\frac{\pi}{2 m}\right)}+\frac{m}{\pi} \log \cot \frac{\pi}{2 m} \leq \frac{m}{\pi} \log (5 m),
\end{aligned}
$$

which completes the proof of (8).

2.2. Proof of Theorem 1, Let $1 \leq M<N \leq p-1$. Using (7) with $A=M^{\prime}:=$ $[M / 2]$ and $B=N^{\prime}:=[N / 2]$, for $1 \leq n \leq p-1$ we have

$$
(-1)^{n} \mathbf{1}_{\left[2 M^{\prime}+1,2 N^{\prime}\right]}(n)=\frac{1}{p} \sum_{1 \leq|h| \leq(p-1) / 2} v_{p}\left(h ; M^{\prime}, N^{\prime}\right) \exp (2 \pi i h n / p) .
$$

Similarly, using (7) with $A=0, B=(p-1) / 2$, for $1 \leq n \leq p-1$ we have

$$
(-1)^{n^{*}}=\frac{1}{p} \sum_{1 \leq|k| \leq(p-1) / 2} v_{p}(k ; 0,(p-1) / 2) \exp \left(2 \pi i k n^{*} / p\right) .
$$


Hence, since $2 M^{\prime} \leq M \leq 2 M^{\prime}+1$ and $2 N^{\prime} \leq N \leq 2 N^{\prime}+1$,

$$
\begin{aligned}
& \sum_{M \leq n \leq N} e_{n}=\sum_{1 \leq n \leq p-1}(-1)^{n}(-1)^{n^{*}} \mathbf{1}_{\left[2 M^{\prime}+1,2 N^{\prime}\right]}(n)+2 \theta(M, N) \\
& =\frac{1}{p^{2}} \sum_{1 \leq|h|,|k| \leq(p-1) / 2} v_{p}\left(h ; 0, N^{\prime}\right) v_{p}(k ; 0,(p-1) / 2) S(h, k)+2 \theta(M, N),
\end{aligned}
$$

where $|\theta(M, N)| \leq 1$ and $S(h, k)$ is a Kloosterman's sum:

$$
S(h, k)=\sum_{1 \leq n \leq p-1} \exp \left(2 \pi i\left(h n+k n^{*}\right) / p\right) .
$$

By Weil's theorem [10], we have $|S(h, k)| \leq 2 \sqrt{p}$ whenever $(h, p)=1$ or $(k, p)=1$. This last condition is always satisfied. Therefore, applying (8) twice, we complete the proof of Theorem 1 .

Remark 4. Further pseudorandom properties of Kloosterman's sums can be found in É. Fouvry, P. Michel, J. Rivat, A. Sárközy [4].

\section{Proof of Theorem 2}

3.1. Exponential sums. We will use exponential sums which can be handled by Weil's theorem [10]. In the special case of interest for us, a convenient form was given by C.J. Moreno and O. Moreno [8, Theorem 2]. Using some arguments of J. Eichenauer-Herrmann and H. Niederreiter [3, p. 272], we proved the following.

Lemma 5. Let $p$ be a prime number and $Q / R$ a rational function over $\mathbf{F}_{p}$, which is not constant. Let $s$ be the number of distinct roots of the polynomial $R$ in $\overline{\mathbf{F}_{p}}$. If $\psi$ is a non-trivial additive character of $\mathbf{F}_{p}$, then

$$
\left|\sum_{\substack{n \in \mathbf{F}_{p} \\ R(n) \neq 0}} \psi\left(\frac{Q(n)}{R(n)}\right)\right| \leq(\max (\operatorname{deg}(Q), \operatorname{deg}(R))+s-1) \sqrt{p} .
$$

Proof. This is Lemma 13 of $[9]$.

Lemma 6. Let $A, B, m \in \mathbf{Z}_{\geq 0}$ such that $m \geq 3$ is odd and $0 \leq A<B \leq m-1$. Then, for $n \in \mathbf{Z}$ with $1 \leq n \leq m-1$, we have

$$
\mathbf{1}_{[A+1, B]}(n)=\frac{1}{m} \sum_{|h| \leq(m-1) / 2} u_{m}(h ; A, B) \exp (2 \pi i h n / m),
$$

where $u_{m}(0, A, B)=B-A$ and $u_{m}(h ; A, B)$ is defined for $0<|h| \leq(m-1) / 2$ by

$$
\begin{aligned}
u_{m}(h ; A, B) & =\sum_{A+1 \leq k \leq B} \exp \left(\frac{-2 \pi i h k}{m}\right) \\
& =\frac{\sin (\pi h(B-A) / m)}{\sin (\pi h / m)} \exp \left(\frac{-\pi i h}{m}(A+B+1)\right),
\end{aligned}
$$

and satisfies

$$
\sum_{|h| \leq(m-1) / 2}\left|u_{m}(h ; A, B)\right| \leq B-A+\frac{2 m}{\pi} \log \frac{4 m}{\pi} .
$$


Proof. For odd $m \geq 3$ and $1 \leq n \leq m-1$, we have

$$
\begin{aligned}
\mathbf{1}_{[A+1, B]}(n) & =\sum_{A+1 \leq k \leq B} \mathbf{1}_{\{k\}}(n)=\sum_{A+1 \leq k \leq B} \frac{1}{m} \sum_{|h| \leq(m-1) / 2} \exp \left(\frac{2 \pi i h(n-k)}{m}\right) \\
& =\frac{1}{m} \sum_{|h| \leq(m-1) / 2} u_{m}(h ; A, B) \exp (2 \pi i h n / m),
\end{aligned}
$$

which proves (91). To prove (10), it suffices to observe that

$$
\left|u_{m}(h ; A, B)\right| \leq 1 / \sin (\pi|h| / m)
$$

for $0<|h| \leq(m-1) / 2$. Therefore

$$
\sum_{|h| \leq(m-1) / 2}\left|u_{m}(h ; A, B)\right| \leq B-A+2 \sum_{1 \leq h \leq(m-1) / 2} \frac{1}{\sin (\pi h / m)}
$$

and by the convexity of $t \mapsto 1 / \sin (\pi t / m)$, we have

$$
\sum_{1 \leq h \leq(m-1) / 2} \frac{1}{\sin (\pi h / m)} \leq \int_{1 / 2}^{m / 2} \frac{d t}{\sin (\pi t / m)}=\frac{m}{\pi} \log \cot \frac{\pi}{4 m} \leq \frac{m}{\pi} \log \frac{4 m}{\pi},
$$

which completes the proof of (10).

3.2. Proof of Theorem 2, Let $1 \leq t<p$ and $g(j):=f(a+j b)$. Using (7) with $A=0$ and $B=(p-1) / 2$, and writing $v_{p}(l)=v_{p}(l ; 0,(p-1) / 2)$, for $j$ such that $g(j) \neq 0$ we have

$$
\begin{aligned}
(-1)^{g(j)} & =\frac{1}{p} \sum_{1 \leq|h| \leq(p-1) / 2} v_{p}(h) \exp (2 \pi i h g(j) / p) \\
(-1)^{g(j)^{*}} & =\frac{1}{p} \sum_{1 \leq|k| \leq(p-1) / 2} v_{p}(k) \exp \left(2 \pi i k g(j)^{*} / p\right) .
\end{aligned}
$$

Furthermore, using (9) with $A=0$ and $B=[(t-1) / 2]$, and writing $u_{p}(l)=$ $u_{p}(l ; 0,[(t-1) / 2])$, there exist $\theta_{t} \in \mathbf{Z}$ with $\left|\theta_{t}\right| \leq 1$ such that

$$
\mathbf{1}_{[1, t-1]}(j)=\frac{1}{p} \sum_{|l| \leq(p-1) / 2} u_{p}(l) \exp (2 \pi i l j / p)+\theta_{t} .
$$

Hence, we get

$$
\sum_{\substack{0 \leq j \leq t-1 \\ g(j) \neq 0}} e_{a+j b}=\frac{1}{p^{3}} \sum_{\substack{|l| \leq(p-1) / 2 \\ \sum_{1 \leq|h|,|k| \leq(p-1) / 2}}} u_{p}(l) v_{p}(h) v_{p}(k) S(h, k, l)+\theta_{t}^{\prime},
$$

where $\left|\theta_{t}^{\prime}\right| \leq 1$ and $S(h, k, l)$ is a sort of generalized Kloosterman's sum:

$$
S(h, k, l)=\sum_{\substack{0 \leq j \leq-1 \\ g(j) \neq 0}} \exp \left(2 \pi i\left(l j+h g(j)+k g(j)^{*}\right) / p\right) .
$$

Since $0<b<p$, the polynomial $g(x)$ is of degree $d=\operatorname{deg} f$, and has $s$ distinct roots in $\overline{\mathbf{F}_{p}}$. Therefore, by Lemma 5 , we have for $1 \leq|k| \leq(p-1) / 2$,

$$
|S(h, k)| \ll(d+s) \sqrt{p},
$$


and by (10) and (8), we get

$$
\left|\sum_{\substack{0 \leq j \leq t-1 \\ g(j) \neq 0}} e_{a+j b}\right| \ll(d+s) p^{1 / 2} \log ^{3} p .
$$

Furthermore, there are at most $s$ values of $j$ such that $g(j)=f(a+j b)=0$, and their contribution is negligible since $s \leq(d+s) p^{1 / 2} \log ^{3} p$. This completes the proof of (5).

The proof of (6) is similar. By the same arguments with $H=(p-1) / 2$, we can write

$$
\begin{aligned}
& \sum_{\substack{1 \leq n \leq M \\
f\left(n+d_{1}\right) f\left(n+d_{2}\right) \neq 0}} e_{n+d_{1}} e_{n+d_{2}} \\
& =\frac{1}{p^{5}} \sum_{\substack{|l| \leq H \\
\\
\quad}} \sum_{1 \leq\left|h_{1}\right|,\left|h_{2}\right|,\left|k_{1}\right|,\left|k_{2}\right| \leq H} u_{p}(l) v_{p}\left(h_{1}\right) v_{p}\left(h_{2}\right) v_{p}\left(k_{1}\right) v_{p}\left(k_{2}\right) T\left(h_{1}, h_{2}, k_{1}, k_{2}, l\right)
\end{aligned}
$$

where

$$
T\left(h_{1}, h_{2}, k_{1}, k_{2}, l\right)=\sum_{\substack{0 \leq n \leq p-1 \\ f\left(n+d_{1}\right) f\left(n+d_{2}\right) \neq 0}} \exp (2 \pi i P(n) / p)=\sum_{\substack{0 \leq n \leq p-1 \\ R(n) \neq 0}} \exp \left(2 \pi i \frac{Q(n)}{R(n)} / p\right),
$$

with

$$
\begin{aligned}
P(n)= & l n+h_{1} f\left(n+d_{1}\right)+h_{2} f\left(n+d_{2}\right)+k_{1} f\left(n+d_{1}\right)^{*}+k_{2} f\left(n+d_{2}\right)^{*}, \\
Q(n)= & \left(l n+h_{1} f\left(n+d_{1}\right)+h_{2} f\left(n+d_{2}\right)\right) f\left(n+d_{1}\right) f\left(n+d_{2}\right) \\
& +k_{1} f\left(n+d_{2}\right)+k_{2} f\left(n+d_{1}\right), \\
R(n)= & f\left(n+d_{1}\right) f\left(n+d_{2}\right) .
\end{aligned}
$$

In order to complete the proof of (6), using (10) and (8), it remains to prove, for all $h_{1}, h_{2}, k_{1}, k_{2}, l$ with $1 \leq\left|h_{1}\right|,\left|h_{2}\right|,\left|k_{1}\right|,\left|k_{2}\right| \leq H$ and $|l| \leq H$, the estimate:

$$
\left|T\left(h_{1}, h_{2}, k_{1}, k_{2}, l\right)\right| \ll(d+s) \sqrt{p} \text {. }
$$

We may assume that $p \geq 11$ and $d=\operatorname{deg} f \leq \sqrt{p}$, for otherwise (6) is trivial. This ensures that $\max (\operatorname{deg} Q, \operatorname{deg} R)<p$. Then we distinguish two cases:

- If $n \mapsto l n+h_{1} f\left(n+d_{1}\right)+h_{2} f\left(n+d_{2}\right)$ is not constant, then $0<\operatorname{deg} R<$ $\operatorname{deg} Q<p$ and the rational function $Q / R$ over $\mathbf{F}_{p}$ is not constant, so that by Lemma 5 we get (11).

- If $n \mapsto \ln +h_{1} f\left(n+d_{1}\right)+h_{2} f\left(n+d_{2}\right)$ is constant, then we must estimate

$$
\sum_{R(n) \neq 0} \exp \left(2 \pi i\left(\frac{k_{1} f\left(n+d_{2}\right)+k_{2} f\left(n+d_{1}\right)}{f\left(n+d_{1}\right) f\left(n+d_{2}\right)}\right) / p\right) \text {. }
$$

If the numerator is a polynomial $\neq 0$, then the denominator is of higher degree than the numerator, and again by Lemma 5 we get (11). But if the numerator $=0$, then considering the coefficients of higher degree we must have $k_{2}=-k_{1}$, and then $d_{1} \equiv d_{2} \bmod p$, which is impossible since $0 \leq d_{1}<d_{2}<p$.

We remark that the correlation of order $k>2$ can be expressed by a similar formula. Again, the estimate of the innermost sum leads to the estimate of an 
exponential sum involving a rational function, i.e., of the type occuring in Lemma 5. However, in order to be able to use Lemma 5] we need the fact that this rational function is not constant. If $k>2$, then to ensure this one needs further assumptions as in [7] on $f(n)$ and a lengthy discussion; here we do not go into the details of this.

\section{REFERENCES}

[1] N. Alon, Y. Kohayakawa, C. Mauduit, C.-G. Moreira, and V. Rödl, Measures of pseudorandomness for finite sequences: minimum and typical values, submitted to Combinatorics, Probability and Computation (to appear).

[2] Julien Cassaigne, Christian Mauduit, and András Sárközy, On finite pseudorandom binary sequences VII: The measures of pseudorandomness, Acta Arithmetica 103 (2002), no. 2, 97-118. MR.1904866 (2004c:11139)

[3] Jürgen Eichenauer-Herrmann and Harald Niederreiter, Bounds for exponential sums and their applications to pseudorandom numbers, Acta Arithmetica 67 (1994), no. 3, 269-281. MR1292739 (95g:11079)

[4] Etienne Fouvry, Philippe Michel, Joël Rivat, and András Sárközy, On the pseudorandomness of the signs of Kloosterman sums, Journal of the Australian Mathematical Society 77 (2004), 425-436. MR2099811 (2005h:11165)

[5] Richard K. Guy, Unsolved problems in number theory, Unsolved Problems in Intuitive Mathematics, vol. 1, Springer-Verlag, New York, 1981, Problem Books in Mathematics. MR 0656313 (83k:10002)

[6] Christian Mauduit and András Sárközy, On finite pseudorandom binary sequences I: Measure of pseudorandomness, the Legendre symbol, Acta Arithmetica 82 (1997), 365-377. MR.1483689 (99g:11095)

[7] Christian Mauduit and András Sárközy, Construction of pseudorandom binary sequences by using the multiplicative inverse, Acta Mathematica Hungarica 108 (2005), 239-252. MR.2162562(2006c:11092)

[8] C. J. Moreno and O. Moreno, Exponential sums and Goppa codes: I, Proceedings of the American Mathematical Society 111 (1991), 523-531. MR.1028291 (91f:11087)

[9] Joël Rivat and András Sárközy, Modular constructions of pseudorandom binary sequences with composite moduli, Periodica Mathematica Hungarica 51 (2005), 75-107. MR2194941

[10] A. Weil, Sur les courbes algébriques et les variétés qui s'en déduisent, Act. Sci. Ind., vol. 1041, Hermann, Paris, 1948. MR.0027151(10:262c)

[11] Zhang Wenpeng, On a problem of D. H. Lehmer and its generalization, Compositio Math. 86 (1993), no. 3, 307-316. MR.1219630 (94f:11104)

[12] Wen Peng Zhang, A problem of D. H. Lehmer and its generalization. II, Compositio Math. 91 (1994), no. 1, 47-56. MR1273925 (95f:11079)

[13] Yi Yuan and Wen-peng Zhang, On generalization of Lehmer D H problem, Gongcheng Shuxue Xuebao 20 (2003), no. 1, 60-64. MR1975320(2004b:11123)

[14] Wenpeng Zhang, A problem of D. H. Lehmer and its mean square value formula, Japan. J. Math. (N.S.) 29 (2003), no. 1, 109-116. MR1986866 (2004d:11097)

[15] Wenpeng Zhang, A problem of D. H. Lehmer and Kloosterman's sums, Monatshefte für Mathematik 139 (2003), 247-257. MR1994384(2004e:11088)

[16] Wenpeng Zhang, Xu Zongben, and Yi Yuan, A problem of D. H. Lehmer and its mean square value formula, J. Number Theory 103 (2003), no. 2, 197-213. MR.2020268 (2004m:11128)

Institut de Mathématiques de Luminy, UMR 6206, 163, avenue de Luminy, Case 907, 13288 Marseille Cedex 9, France

E-mail address: loubouti@iml.univ-mrs.fr

Institut de Mathématiques de Luminy, UMR 6206, 163, avenue de Luminy, Case 907, 13288 Marseille Cedex 9, France

E-mail address: rivat@iml.univ-mrs.fr

Department of Algebra and Number Theory, Eötvös Loránd University, H-1117 BuDAPEST, PÁzmány PÉter SÉtány 1/C, Hungary

E-mail address: sarkozy@cs.elte.hu 